Abanico Veterinario. Enero-Febrero 2020; 10:1-11. http://dx.doi.org/10.21929/abavet2020.20 Nota corta. Recibido: 02/05/2020. Aceptado: 03/08/2020. Publicado: 17/08/2020. Clave: 2020-59.

\title{
Evaluación del desprendimiento de oocistos de Eimeria maxima y Eimeria acervulina en pollos de engorde
}

\author{
Evaluation of oocyst shedding of Eimeria maxima and Eimeria acervulina in broiler \\ chickens
}

\begin{abstract}
Lauren Laverty ${ }^{1 \mathrm{ID}}$, Roberto Señas-Cuesta ${ }^{1 \mathrm{ID}}$, Sergio Martínez-González ${ }^{\star 2} \mathrm{ID}$, Callie Selby ${ }^{1 \text { ID }}$, Guillermo Tellez-Jr ${ }^{1 \text { ID }}$, Xochitl Hernandez-Velasco ${ }^{3}$ ID , Christine Vuong ${ }^{1 \text { ID }}$ Billy Hargis $^{1 \text { ID }}$, Guillermo Tellez-Isaias ${ }^{1 \text { ID }}$, Danielle Graham ${ }^{1 \text { ID }}$
\end{abstract}

1'Department of Poultry Science, University of Arkansas Division of Agriculture, Fayetteville, AR 72701. ${ }^{2}$ Unidad Académica de Medicina Veterinaria y Zootecnia. Universidad Autónoma de Nayarit. México. ${ }^{3}$ Departamento de Medicina y Zootecnia de Aves, Facultad de Medicina Veterinaria y Zootecnia, UNAM 04510, México. *Autor para correspondencia: Universidad Autónoma de Nayarit, Unidad Académica de Medicina Veterinaria y Zootecnia. Km 3.5 Carretera Compostela-Chapalilla. Compostela, Nayarit, México. CP.63700.Imlavert@uark.edu, rsenascu@uark.edu, sergio.martinez@uan.edu.mx, mccreer@uark.edu, memotellez98@gmail.com, xochitl_h@yahoo.com, vuong@uark.edu, bhargis@uark.edu, gtellez@uark.edu, bmahaffe@uark.edu

\section{RESUMEN}

El propósito del presente estudio fue evaluar el día y la hora de recolección de la muestra de un desafío experimental con Eimeria maxima (EM) y Eimeria acervulina (EA) en pollos de engorde. Los pollos de engorde macho Cobb-Vantress de un día de edad se asignaron aleatoriamente a uno de tres grupos con diez réplicas ( $\mathrm{n}=8$ pollos / réplica). Los pollos se colocaron en jaulas de batería con un entorno controlado apropiado para la edad: Grupo 1) Control negativo (sin desafío o tratamiento); 2) Control de desafío (solo desafío Eimeria); 3) Desafío + salinomicina. A los 14 días de edad, los pollos fueron desafiados por vía oral con el cultivo mixto de EM/EA (10,000 EM esporulados que contenían 4\% de EA de tipo salvaje). Los parámetros de rendimiento se registraron en los días 7, 14, 20 y 23. Los puntajes de las lesiones se registraron post mortem en los días 20 y 23. El oocisto por gramo (OPG) se realizó en los días seis, siete y ocho después del desafío, y muestras se recolectaron a las 9:00 a.m y a las 6:00 p.m. de cada día, respectivamente. Los recuentos de oocistos fueron significativamente diferentes $(P<0.05)$ entre la mañana y la tarde en el día seis después del desafío con coccidios. Los resultados de este estudio muestran que el día y la hora en que se recolectan las muestras pueden tener un efecto significativo en la confiabilidad y validez de los datos.

Palabras clave: Eimeria maxima; Eimeria acervulina; desprendimiento de oocistos; parámetros de rendimiento.

The purpose of the present study was to evaluate the day and the time of sample collection of an experimental challenge with Eimeria maxima (EM) and Eimeria acervulina (EA) in broiler chickens. Oneday old male Cobb-Vantress broiler chickens were randomly allocated to one of three groups with ten replicates ( $n=8$ chickens/replicate). Chickens were placed in battery cages with a controlled age-appropriate environment: Group 1) Negative control (no challenge or treatment); 2) Challenge control (Eimeria challenge only); 3) Challenge + Salinomycin. Challenged chickens were orally gavaged with the mixed culture of EM/EA (10,000 sporulated EM containing 4\% wild-type EA) at 14 days of age. Performance parameters were recorded at days 7, 14, 20, and 23. Lesions scores were recorded post-mortem on days 20 and 23. Oocyst per gram (OPG) was performed on days six, seven, and eight post-challenge, and samples were collected at 9:00 AM and 6:00 PM on each day, respectively. Oocyst counts were significantly different ( $P$ $<0.05$ ) between morning and afternoon on day six post coccidia challenge. The results of this study show 
that the day and the time at which samples are collected can have a significant effect on the reliability and validity of data.

Keywords: Eimeria maxima; Eimeria acervulina; oocysts shedding; performance parameters.

\section{INTRODUCCIÓN}

La coccidiosis actualmente demuestra ser una enfermedad protozoaria importante y urgente en la industria avícola en todo el mundo (Dalloul y Lillehoj, 2006). La coccidiosis es causada por un parásito protozoario del género Eimeria. El ciclo de vida de los parásitos coccidiales incluye etapas de replicación asexual y sexual y comienza cuando un ave ingiere oocistos esporulados del medio ambiente, como describen Conway y McKenzie (2007) Después de la ingestión, cuatro esporoquistes contenidos en un solo oocisto esporulado liberan dos esporozoitos. La liberación de los esporozoitos es causada por la actividad digestiva dentro del pollo. Los esporozoitos liberados "invadirán las células epiteliales en una zona específica del intestino o ceca"; que depende de la especie Eimeria (Chapman, 2003). Dentro de la célula, los esporozoítos se convierten en trofozoítos y se alimentan durante 12 a 48 para crecer y finalmente dividirse asexualmente a través de esquizogonia o merogonía; esta etapa se conoce como esquizonte o meront. Dentro del parásito, las etapas de merozoito se forman y se liberan después de que el esquizonte madura y se rompe, lo que toma tres días. Esta primera generación de merozoitos invadirá más células epiteliales y repetirá el proceso de multiplicación. La segunda generación de merozoitos puede inducir un tercer ciclo esquizogónico; esto también depende de la especie Eimeria. Se formarán gametocitos masculinos (microgametocitos) y femeninos (macrogametocitos). Los macrogametocitos se convertirán en macrogametos. Los microgametocitos madurarán, se romperán y liberarán microgametos biflagelados que fertilizan a los macrogametos femeninos. Después de la fertilización, "se forma una pared engrosada alrededor del macrogameto, formando un cigoto" (Conway y McKenzie, 2007; McDougald and Fitz-Coy, 2013). Al final de este ciclo, se forma un nuevo ooquiste que pasará a través de los excrementos del ave después de romper su célula huésped (Tewari y Maharana, 2011).

Los oocistos de Eimeria spp, de una o varias infecciones simultáneas, se excretan en las heces durante un período de varios días. La excreción de oocistos comienza en un nivel bajo, alcanza una meseta y luego disminuye hasta que la enfermedad sigue su curso (Clarke, 1979; Williams, 1973). Curiosamente, varios investigadores han informado que los recuentos de oocistos difieren entre las recolecciones de muestreo matutino y vespertino (Hudman et al., 2000; Brown et al., 2001). Esta variabilidad se ha reconocido durante varios años, pero en gran medida se ha pasado por alto (Misof, 2004). Sin embargo, recientemente se ha demostrado que el día posterior a la inoculación y la hora del día en que se recolectan las muestras pueden tener un efecto significativo en la confiabilidad y validez de los datos (Brawner y Hill, 1999). Por lo tanto, el propósito del presente estudio fue evaluar la influencia de la variación en la excreción de oocistos y el 
día de muestreo sobre el desafío experimental con Eimeria maxima y Eimeria acervulina en pollos de engorde.

\section{Cepas de desafío}

\section{MATERIAL Y MÉTODOS}

El Dr. John R. Barta proporcionó oocistos de Eimeria maxima M6 (EM) y E. acervulina (EA) de tipo salvaje, Universidad de Guelph, Canadá. Los métodos para detectar y recuperar oocistos de pollos infectados, esporulación de oocistos y preparación de dosis infecciosas, se llevaron a cabo como se describió anteriormente (Haug et al., 2006). Se llevó a cabo un estudio de titulación de la dosis para determinar la dosis de co-desafío de coccidia EM/EA antes de comenzar el ensayo experimental. A los 13 días de edad, los pollos de engorde se pesaron, se dividieron en tres grupos ( $n=15 / g r u p o$ ) y se desafiaron con tres dosis diferentes $(10,000,20,000$ o 40,000) de oocistos esporulados en $1 \mathrm{~mL}$ de volumen por sonda oral. El cuarto grupo de pollitos se mantuvo como control negativo. Cinco días después de la exposición, se registraron el peso corporal (PC) y la ganancia de peso corporal (GPC). En el presente estudio, los pollos expuestos fueron alimentados por sonda oral a las 9:00 am con el cultivo mixto de EM/EA (10,000 EM esporulados que contienen $4 \%$ de EA de tipo salvaje) a los 14 días de edad ya que esta dosis redujo el BWG en un $35.82 \%$. Esto se basa en el criterio de que la dosis de provocación debe causar coccidiosis subclínica, que consiste en una reducción entre el 25-35\% del GPC sin la presencia de signos clínicos.

\section{Fuente animal y diseño experimental}

Se pesaron 240 pollos de engorde Cobb-Vantress machos de un día de edad (Fayetteville, AR, EE. UU.) Y se asignaron al azar a uno de tres grupos con diez repeticiones ( $n=8$ pollos/repetición). Los pollos se colocaron en jaulas en batería, con un ambiente controlado apropiado para la edad: Grupo 1) Control negativo (sin desafío ni tratamiento); 2) Control de desafío (solo desafío de Eimeria); 3) Desafío + Salinomicina a $60 \mathrm{~g}$ /tonelada (Bio-Cox 60, Huvepharma, Peachtree City, GA 30269). Los pollitos recibieron acceso ad libitum al agua y al alimento durante 23 días. Se formuló una dieta de iniciación experimental (Tabla 1) para aproximar los requisitos nutricionales de los pollos de engorde según lo recomendado por el Consejo Nacional de Investigación (NRC, 1994) y se ajustó a las recomendaciones de los criadores Cobb, 2015). Los pollos recibieron 23 horas de luz del día primero al cuarto, 20 horas de luz del día 5 al 14 y 18 horas de luz del día 15 al 23. La intensidad de la luz se fijó en una vela de 30 pies la primera semana, una vela de 1 pie de los días ocho a catorce, y una vela de 0.5 pies de los días 15 a 23. La temperatura y la luz se establecieron para imitar las condiciones comerciales del día 1-21 en todas las habitaciones con una reducción gradual de la temperatura de 32 a $24{ }^{\circ} \mathrm{C}$ y la humedad relativa a $55 \pm 5 \%$. Los parámetros de rendimiento: peso corporal (PC), ganancia de peso corporal (GPC), consumo de alimento (CA) e índice de conversión alimenticia (ICA) se registraron los días 7, 14, 20 y 23. El día 20, la mitad de los pollos de cada réplica se pesaron y sacrificaron mientras que los pollos restantes se pesaron y sacrificaron el día 23 para evaluar las lesiones macroscópicas de 
acuerdo con el sistema de puntuación de Johnson y Reid (Johnson y Reid, 1970). Se evaluó el oocisto por gramo (OPG) los días seis, siete y ocho después de la exposición, y las muestras se recolectaron a las 9:00 AM y 6:00 PM todos los días, respectivamente. Todos los procedimientos de manipulación de animales cumplieron con el Comité Institucional de Uso y Cuidado de Animales (IACUC) de la Universidad de Arkansas, Fayetteville. De manera explícita, el IACUC aprobó este estudio bajo el protocolo \# 21020.

Tabla 1. Composición de ingredientes y contenido de nutrientes de una dieta de iniciación de maíz y soja utilizada en todos los grupos experimentales tal cual.

\begin{tabular}{|c|c|}
\hline Ítem & Dieta de inicio \\
\hline \multicolumn{2}{|l|}{ Ingredientes (\%) } \\
\hline Maíz & 57.34 \\
\hline Harina de soja & 34.66 \\
\hline Grasa de ave & 3.45 \\
\hline Fosfato dicálcico & 1.86 \\
\hline Carbonato de calcio & 0.99 \\
\hline sal & 0.38 \\
\hline DL-metionina & 0.33 \\
\hline L-lisina $\mathrm{HCl}$ & 0.31 \\
\hline Treonina & 0.16 \\
\hline Premezcla de vitaminas ${ }^{1}$ & 0.20 \\
\hline Premezcla mineral ${ }^{2}$ & 0.10 \\
\hline Cloruro de colina $60 \%$ & 0.20 \\
\hline Antioxidante $^{3}$ & 0.02 \\
\hline \multicolumn{2}{|l|}{ Análisis calculado } \\
\hline Energía metabolizable (kcal / kg) & 3,035 \\
\hline Proteína cruda (\%) & 22.16 \\
\hline Extracto de éter (\%) & 5.68 \\
\hline Lisina $(\%)$ & 1.35 \\
\hline Metionina (\%) & 0.64 \\
\hline Metionina + cistina $(\%)$ & 0.99 \\
\hline Treonina (\%) & 0.92 \\
\hline Triptófano (\%) & 0.28 \\
\hline Calcio total & 0.90 \\
\hline Fósforo disponible & 0.45 \\
\hline \multicolumn{2}{|l|}{ Análisis determinado } \\
\hline Proteína cruda (\%) & 21.15 \\
\hline Extracto de éter (\%) & 6.05 \\
\hline Calcio (\%) & 0.94 \\
\hline Fósforo (\%) & 0.73 \\
\hline
\end{tabular}




\section{Análisis estadístico y de datos}

Las puntuaciones de las lesiones, los oocistos por gramo y los datos de rendimiento se sometieron a ANOVA como un diseño completamente al azar utilizando el procedimiento GLM de SAS (SAS, 2002). Para los parámetros de rendimiento del crecimiento (PC, GPC, CA y ICA), cada jaula replicada se consideró como una unidad experimental. Las medias del tratamiento se dividieron usando la prueba de rango múltiple de Duncan a $\mathrm{P}<0,05$, lo que indica significación estadística.

\section{RESULTADOS Y DISCUSIÓN}

Los resultados de la evaluación del peso corporal, la ganancia de peso corporal, el consumo de alimento y la índice de conversión alimenticia en pollos de engorde desafiados con coccidios se resumen en la Tabla 2. Los tres grupos comenzaron con un peso corporal similar; sin embargo, en el día 7, hubo un aumento en el peso corporal de los pollos tratados con salinomicina. Para el día 20 (6 días después de la exposición), el grupo de control negativo (no desafiado o tratado) y el grupo tratado con salinomicina de desafío mostraron un aumento significativo en el peso corporal en comparación con el grupo de control desafiado $(P<0.05)$. Curiosamente, el día 23 (9 días después de la exposición), solo hubo diferencias significativas en el peso corporal entre el grupo de control negativo y el control de exposición. Se observó una tendencia similar en GPC e ICA. No se observaron diferencias significativas en CA entre los tres grupos (Tabla 2).

La Tabla 3 muestra los resultados de la evaluación del recuento de oocistos de E. maxima por gramo en las heces de pollos de engorde desde el día 6 hasta el día 8 después de la exposición a diferentes momentos del día, el promedio por día. Aunque hubo cierta recuperación de oocistos de pollos de control no tratados y no desafiados, hubo significativamente menos OPG en este grupo en comparación con ambos grupos de desafío. No se observaron diferencias significativas en OPG entre ambos grupos de desafío durante los tres días de evaluación (Tabla 3). En el presente estudio, fue notable encontrar que los oocistos EM se excretaron en cantidades muy altas el día 6 después de la exposición por la noche para los tres grupos experimentales. Al combinar y obtener la OPG promedio, el día 6 mostró un mayor número de oocistos EM, y se observaron las diferencias significativas esperadas entre las OPG entre los tres grupos experimentales (Tabla 3). De manera similar, se encontraron diferencias significativas en las puntuaciones de lesiones para EM para ambos días de evaluación (20 y 23 días) entre los tres grupos experimentales. No obstante, se recuperó un mayor número de oocistos el día 20 en ambos grupos desafiados en comparación con el día 23 (Tabla 3). En el presente estudio, los pollos de control negativo fueron asignados aleatoriamente a los grupos experimentales que fueron desafiados con coccidios. Quizás, esa es la razón por la que estos pollos mostraron alguna infección, debido a la contaminación cruzada de heces entre las jaulas. Claramente, en estudios futuros, los pollos de control negativo deben colocarse en una habitación separada y si esto no es posible, en jaulas separadas y aisladas. 
Los resultados de la evaluación de oocistos de E. acervulina por recuento de gramos en las heces de pollos de engorde del día 6 al día 8 después de la exposición en diferentes momentos del día, el promedio por día y las puntuaciones de lesiones en los días 20 y 23 se resumen en Tabla 4. Se observó una tendencia similar en la OPG para EA, aunque hubo más oocistos en el día 23 en los grupos desafiados que en el día 20 (Tabla 4). Las puntuaciones de las lesiones intestinales macroscópicas en los días 20 y 23 de edad se muestran en la tabla 5. En resumen, los recuentos de oocistos fueron significativamente diferentes entre la mañana y la noche del día 6 después de la exposición. El aumento de la eliminación de oocistos en las recolecciones de muestras vespertinas está de acuerdo con estudios anteriores sobre la excreción diurna de oocistos de Eimeria spp. (Hudman et al., 2000; Brown et al., 2001; Misof, 2004).

La coccidiosis sigue siendo una de las enfermedades más críticas en las aves de corral y resulta en la pérdida anual de millones de dólares estadounidenses por parte de la industria avícola (Williams, 2005; Chapman, 1999). Una práctica común en el manejo de la coccidiosis es el uso de fármacos profilácticos y antimicrobianos que inhiben el desarrollo de esporozoitos/merozoitos. Sin embargo, la industria avícola ahora está experimentando una creciente resistencia a los medicamentos en las cepas de Eimeria (Abbas et al., 2012). Por lo tanto, la presión de la disminución de la eficacia química ha aumentado la demanda de nuevos métodos de tratamiento, como los productos vegetales. Dado que existen claras ventajas de un agente de control eficaz sin complicaciones con la resistencia a los fármacos de Eimeria, es conveniente buscar métodos eficaces con mecanismos alternativos a la quimioterapia anticoccidial tradicional (Naidoo et al., 2008; Masood et al., 2013). La vacunación contra la coccidiosis es una alternativa al uso de productos químicos. Al vacunar contra la coccidiosis, el sistema inmunológico natural del animal se emplea para combatir posibles infecciones en el futuro (Shivaramaiah et al., 2014). De manera convencional, se utilizan parásitos vivos o atenuados y las vacunas específicas de Eimeria pueden incorporar múltiples especies 0 cepas (Shivaramaiah et al., 2014). Los parásitos atenuados de Eimeria se pueden seleccionar a través de la "precocidad", en la que "cepas virulentas de Eimeria sensibles a los fármacos" se les permite pasar a través de una especie hospedadora, reproducirse y convertirse en vacunas atenuadas (Peek y Landman, 2011; Shirley et al., 2007).

Investigaciones anteriores han descrito la variación circadiana en la diseminación de oocistos en múltiples especies de aves hospedadoras (Hudman et al., 2000; Brown et al., 2001). En consecuencia, si no se tiene en cuenta la variación circadiana en la eliminación de oocistos, los resultados de tales pruebas no son confiables y pueden inducir a error (Misof, 2004). Un método adecuado para obtener datos precisos parece ser restringir el período de muestreo.

Los resultados de este estudio muestran que el día y la hora en que se recolectan las muestras pueden tener un impacto significativo en los datos y refuerzan la importancia 
de recolectar las muestras fecales a la misma hora del día después del desafío. Los recuentos de oocistos fueron significativamente diferentes entre la mañana y la tarde del día seis después de la exposición a coccidios. El muestreo de carga de coccidios debe restringirse a la segunda mitad del tiempo total de luz diurna. Por tanto, este período más restrictivo debe considerarse el período preferido para obtener información fiable.

Tabla 2. Evaluación del peso corporal, la ganancia de peso corporal, la ingesta de alimento y el índice de conversión alimenticia en pollos de engorde desafiados con coccidios.

\begin{tabular}{|c|c|c|c|}
\hline Item & $\begin{array}{l}\text { Control negativo (sin } \\
\text { desafío ni tratamiento) }\end{array}$ & $\begin{array}{l}\text { Control de desafío } \\
\text { (solo desafío de } \\
\text { Eimeria) }\end{array}$ & $\begin{array}{c}\text { Salinomicina sódica } \\
\text { tratada con desafío + } \\
(60 \mathrm{~g} / \text { ton })\end{array}$ \\
\hline $\begin{array}{l}\text { Peso corporal }(\mathrm{g}) \\
\text { d } 0 \\
\text { d } 7 \\
\text { d } 14 \\
\text { d } 20 \\
\text { d } 23\end{array}$ & $\begin{array}{c}46.60 \pm 0.19 \\
145.05 \pm 2.16 \mathrm{ba} \\
401.30 \pm 7.28 \mathrm{bc} \\
736.14 \pm 11.23 \mathrm{a} \\
927.51 \pm 20.06^{a}\end{array}$ & $\begin{array}{c}46.16 \pm 0.36 \\
144.48 \pm 2.51 \text { ba } \\
402.63 \pm 9.21 \text { bac } \\
671.97 \pm 16.35^{b} \\
781.25 \pm 42.28^{b}\end{array}$ & $\begin{array}{c}46.70 \pm 0.42 \\
148.95 \pm 2.33 \mathrm{a} \\
429.16 \pm 5.11^{\mathrm{a}} \\
751.09 \pm 8.00^{\mathrm{a}} \\
881.38 \pm 23.87^{\mathrm{ba}}\end{array}$ \\
\hline $\begin{array}{l}\text { Ganancia de peso } \\
\text { corporal }(\mathrm{g}) \\
\text { d } 0 \text { to } 7 \\
\text { d } 7 \text { to } 14 \\
\text { d } 14 \text { to } 20 \\
\text { d } 0 \text { to } 23\end{array}$ & $\begin{array}{c}98.45 \pm 2.13 \mathrm{ba} \\
256.26 \pm 6.15 \\
334.84 \pm 5.67 \mathrm{a} \\
890.31 \pm 29.18 \mathrm{a}\end{array}$ & $\begin{array}{c}98.31 \pm 2.54 \text { ba } \\
258.16 \pm 7.55 \\
269.34 \pm 11.69 \text { b } \\
735.65 \pm 42.60^{b}\end{array}$ & $\begin{array}{l}102.25 \pm 2.36{ }^{a} \\
280.21 \pm 3.82{ }^{a} \\
321.93 \pm 5.47{ }^{a} \\
834.03 \pm 23.61\end{array}$ \\
\hline $\begin{array}{l}\text { Ingesta de alimento } \\
\text { (g) } \\
\text { d } 0 \text { to } 14 \\
\text { d } 0 \text { to } 20 \\
\text { d } 0 \text { to } 23\end{array}$ & $\begin{array}{c}609.89 \pm 12.51 \\
930.19 \pm 37.87 \\
1323.79 \pm 31.08\end{array}$ & $\begin{array}{c}621.93 \pm 16.27 \\
909.81 \pm 36.98 \\
1198.68 \pm 57.19\end{array}$ & $\begin{array}{c}641.06 \pm 15.45 \\
770.41 \pm 30.53 \\
1325.55 \pm 29.11\end{array}$ \\
\hline $\begin{array}{l}\text { Índice de conversión } \\
\text { alimenticia (ajustada) } \\
\text { d } 0 \text { to } 14 \\
\text { d } 0 \text { to } 20 \\
\text { d } 0 \text { to } 23\end{array}$ & $\begin{array}{c}1.50 \pm 0.03 \\
1.44 \pm 0.01 b \\
1.41 \pm 0.06 b\end{array}$ & $\begin{array}{l}1.53 \pm 0.03 \\
1.49 \pm 0.02 \\
1.54 \pm 0.03\end{array}$ & $\begin{array}{l}1.50 \pm 0.04 \\
1.45 \pm 0.02 \\
1.51 \pm 0.03\end{array}$ \\
\hline
\end{tabular}

Los pollos se expusieron a Eimeria maxima (M6) y Eimeria acervulina (tipo salvaje) mediante sonda oral a los 14 días. ${ }^{\mathrm{a}-\mathrm{c}}$ Los valores medios en la misma fila que no comparten una letra común difieren significativamente $(P<0.05)$. Cada valor representa la media \pm error estándar. Diez repeticiones, $\mathrm{n}=8$.

\section{CONCLUSIONES}

Los resultados de este estudio muestran que el día y la hora en que se recolectan las muestras pueden tener un impacto significativo en los datos y refuerzan la importancia de recolectar las muestras fecales a la misma hora del día después del desafío. El muestreo de carga de coccidios debe restringirse a la segunda mitad del tiempo total de luz diurna. Por tanto, este período más restrictivo debe considerarse el período preferido para obtener información fiable. 
Tabla 3. Evaluación de oocistos de E. maxima por recuento de gramo ${ }^{1}$ en las heces de pollos de engorde del día 6 al día 8 después de la exposición en diferentes momentos del día y promedio por día.

\begin{tabular}{|c|c|c|c|c|c|c|c|c|c|}
\hline Tratamiento & $\begin{array}{c}\text { Día } 6 \text { / 9:00 } \\
\text { AM } \\
\text { (día 20) }\end{array}$ & $\begin{array}{c}\text { Día 6 / 6:00 } \\
\text { PM } \\
\text { (día 20) }\end{array}$ & $\begin{array}{c}\text { Día } 6 \\
\text { Promedio } \\
\text { AM/PM }\end{array}$ & $\begin{array}{c}\text { Día } 7 \text { / 9:00 } \\
\text { AM } \\
\text { (día 21) }\end{array}$ & $\begin{array}{c}\text { Día } 7 \text { / 6:00 } \\
\text { PM } \\
\text { (día 21) }\end{array}$ & $\begin{array}{c}\text { Día } 7 \\
\text { Promedio } \\
\text { AM/PM }\end{array}$ & $\begin{array}{c}\text { Día } 8 \text { / 9:00 } \\
\text { AM } \\
\text { (día 22) }\end{array}$ & $\begin{array}{c}\text { Día } 8 \text { / 6:00 } \\
\text { PM } \\
\text { (día 22) }\end{array}$ & $\begin{array}{c}\text { Día } 8 \\
\text { Promedio } \\
\text { AM/PM }\end{array}$ \\
\hline $\begin{array}{c}\text { Control } \\
\text { negativo }\end{array}$ & $\begin{array}{c}239 \pm \\
123.12^{\mathrm{bz}}\end{array}$ & $\begin{array}{c}1,880 \pm \\
350.09^{b} y\end{array}$ & $\begin{array}{c}1,059 \pm \\
192.37^{b z}\end{array}$ & $\begin{array}{c}2,214 \pm \\
2,055.28^{\text {b y }}\end{array}$ & $\begin{array}{c}465 \pm \\
164.91^{\mathrm{bz}}\end{array}$ & $\begin{array}{c}1,340 \pm \\
1,080.81 \mathrm{bz}\end{array}$ & $\begin{array}{c}312 \pm \\
247.92^{\mathrm{b} z}\end{array}$ & $\begin{array}{c}504 \pm \\
399.77^{\mathrm{b} z}\end{array}$ & $\begin{array}{c}408 \pm \\
319.28 \text { by }\end{array}$ \\
\hline $\begin{array}{l}\text { Control de } \\
\text { desafío }\end{array}$ & $\begin{array}{c}39,756 \pm \\
8,540.64^{a} y\end{array}$ & $\begin{array}{c}392,859 \pm \\
53742.38^{a y}\end{array}$ & $\begin{array}{c}216,308 \pm \\
26,680.84^{a x}\end{array}$ & $\begin{array}{c}258,783 \pm \\
34093.03 \text { a w }\end{array}$ & $\begin{array}{c}73,803 \pm \\
20,753.83 \text { ax }\end{array}$ & $\begin{array}{c}166,293 \pm \\
24,541.92 \text { a y }\end{array}$ & $\begin{array}{c}39,751 \pm \\
10,808.39 \text { а у }\end{array}$ & $\begin{array}{c}12,844 \pm \\
2,256.07 \text { a z }\end{array}$ & $\begin{array}{c}26,298 \pm \\
5888.63 \text { a z }\end{array}$ \\
\hline $\begin{array}{c}\text { Desafío + } \\
\text { Salinomicina } \\
\text { sódica tratada }\end{array}$ & $\begin{array}{c}28,060 \pm \\
11,708.46^{a} \text { y }\end{array}$ & $\begin{array}{c}304,517 \pm \\
31,024.37^{\text {a y }}\end{array}$ & $\begin{array}{c}166,288 \pm \\
11,708.46 \text { a y }\end{array}$ & $\begin{array}{c}180,752 \pm \\
39,771.21^{a w}\end{array}$ & $\begin{array}{c}86,940 \pm \\
22,231.97^{a x}\end{array}$ & $\begin{array}{c}133,846 \pm \\
39,771.21 \text { a y }\end{array}$ & $\begin{array}{c}23,440 \pm \\
5,199.72^{\mathrm{a} y z}\end{array}$ & $\begin{array}{c}11,966 \pm \\
1,207.11^{\mathrm{a} z}\end{array}$ & $\begin{array}{c}17,703 \pm \\
5199.72 \text { a z }\end{array}$ \\
\hline
\end{tabular}

Tabla 4. Evaluación de oocistos de E. acervulina por recuento de gramo ${ }^{1}$ en las heces de pollos de engorde del día 6 al día 8 después de la exposición en diferentes momentos del día y promedio por día.

\begin{tabular}{|c|c|c|c|c|c|c|c|c|c|}
\hline Tratamiento & $\begin{array}{l}\text { Día 6 / } \\
\text { 9:00 AM } \\
\text { (día 20) }\end{array}$ & $\begin{array}{l}\text { Día 6 / } \\
\text { 6:00 PM } \\
\text { (día 20) } \\
\end{array}$ & $\begin{array}{c}\text { Día } 6 \\
\text { Promedio } \\
\text { AM/PM }\end{array}$ & $\begin{array}{l}\text { Día } 7 \text { / } \\
\text { 9:00 AM } \\
\text { (día 21) }\end{array}$ & $\begin{array}{c}\text { Día } 7 \text { / } \\
\text { 6:00 PM } \\
\text { (día 21) }\end{array}$ & $\begin{array}{c}\text { Día } 7 \\
\text { Promedio } \\
\text { AM/PM } \\
\end{array}$ & $\begin{array}{c}\text { Día } 8 \text { / } \\
\text { 9:00 AM } \\
\text { (día 22) }\end{array}$ & $\begin{array}{c}\text { Día } 8 \text { / } \\
\text { 6:00 PM } \\
\text { (z 22) }\end{array}$ & $\begin{array}{c}\text { Día } 8 \\
\text { Promedio } \\
\text { AM/PM }\end{array}$ \\
\hline $\begin{array}{c}\text { Control } \\
\text { negativo }\end{array}$ & $\begin{array}{c}83 \pm \\
28.07 \text { a y }\end{array}$ & $\begin{array}{c}52 \pm \\
30.20 \text { cy }\end{array}$ & $55 \pm 29.65^{\text {cy }}$ & $0 \pm 0^{\mathrm{cz}}$ & $0 \pm 0^{\mathrm{b} z}$ & $0 \pm 0^{c z}$ & $\begin{array}{c}52 \pm \\
52.08 \text { cy }\end{array}$ & $\begin{array}{c}26 \pm \\
26.25^{c y}\end{array}$ & $\begin{array}{c}52 \pm \\
52.21^{c y}\end{array}$ \\
\hline $\begin{array}{l}\text { Control de } \\
\text { desafío }\end{array}$ & $\begin{array}{c}1,000 \pm \\
319.03 \text { a yz }\end{array}$ & $\begin{array}{c}5,993 \pm \\
995.74 \text { a w }\end{array}$ & $\begin{array}{c}3,497 \pm \\
352.58^{a y}\end{array}$ & $\begin{array}{c}6,656 \pm \\
1924.02 \text { a w }\end{array}$ & $\begin{array}{c}3,007 \pm \\
1266.66 \mathrm{ax}\end{array}$ & $\begin{array}{c}4,831 \pm \\
1580.37^{a} \text { y }\end{array}$ & $\begin{array}{c}1,800 \pm \\
212.01 \text { ab y }\end{array}$ & $\begin{array}{c}779 \pm \\
228.56^{a b z}\end{array}$ & $\begin{array}{c}1,289 \pm \\
182.52^{\text {ba } ~}\end{array}$ \\
\hline $\begin{array}{l}\text { Desafío + } \\
\text { Salinomicina } \\
\text { sódica } \\
\text { tratada }\end{array}$ & $\begin{array}{c}194 \pm \\
54.9 \text { a } z\end{array}$ & $\begin{array}{c}1,849 \pm \\
498.62 \mathrm{bw}\end{array}$ & $\begin{array}{c}1,022 \pm \\
241.84^{\mathrm{by}}\end{array}$ & $\begin{array}{c}2,287 \pm \\
335.90 \mathrm{~b} w\end{array}$ & $\begin{array}{c}1,912 \pm \\
514.33\end{array}$ & $\begin{array}{c}2,099 \pm \\
270.65^{b y}\end{array}$ & $\begin{array}{c}1,339 \pm \\
88.24^{b x}\end{array}$ & $\begin{array}{l}552 \pm \\
99.01 \text { by }\end{array}$ & $\begin{array}{c}945 \pm \\
69.81^{b z}\end{array}$ \\
\hline
\end{tabular}


Tabla 5. Puntuaciones de lesiones intestinales macroscópicas ${ }^{1}$ en los días 20 y 23 de edad para pollos de engorde desafiados con Eimeria acervulina y E. maxima.

\begin{tabular}{|c|c|c|c|c|}
\hline \multirow[t]{2}{*}{ Tratamiento } & \multicolumn{2}{|c|}{ Eimeria acervulina } & \multicolumn{2}{|c|}{ Eimeria maxima } \\
\hline & día 20 & día 23 & día 20 & día 23 \\
\hline Control negativo & $0.00 \pm 0.00^{c}$ & $0.00 \pm 0.00^{c}$ & $0.05 \pm 0.03^{c}$ & $0.00 \pm 0.00^{c}$ \\
\hline Control de desafío & $0.80 \pm 0.12^{a z}$ & $1.45 \pm 0.11$ a y & $1.93 \pm 0.10$ a y & $1.25 \pm 0.08$ a z \\
\hline $\begin{array}{l}\text { Desafío + Salinomicina } \\
\text { sódica tratada }\end{array}$ & $0.48 \pm 0.11^{b z}$ & $0.90 \pm 0.09 \mathrm{~b} y$ & $1.28 \pm 0.14$ by & $0.77 \pm 0.09 b z$ \\
\hline
\end{tabular}

Financiamiento: La investigación fue apoyada en parte por fondos proporcionados por USDA-NIFA Sustainable Agriculture Systems, Grant No. 2019-69012-29905. Título del proyecto: Empoderamiento de la producción de pollos de engorde de EE. UU. Para la transformación y la sostenibilidad USDA-NIFA (Sistemas de agricultura sostenible): No. 2019-69012-29905

Conflictos de interés: Los autores declaran no tener ningún conflicto de intereses.

\section{LITERATURA CITADA}

ABBAS R, Colwell D, Gilleard J. 2012. Botanicals: an alternative approach for the control of avian coccidiosis. World's Poultry Science Journal. 68(2):203-215. https://doi.org/10.1017/S0043933912000268

BRAWNER III WR, Hill GE. 1999. Temporal variation in shedding of coccidial oocysts: implications for sexual-selection studies. Can. J. Zool. 77:347-350.

https://doi.org/10.1139/z98-207

BROWN MA, Ball S, Holman D. 2001. The periodicity of isosporan oocyst discharge in the greenfinch (Carduelis chloris). J. Nat. Hist. 35:945-948. https://doi.org/10.1080/002229301300323875

CHAPMAN HD. 2003. Origins of coccidiosis research in the fowl-The first fifty years. Avian Dis. 47:1-20. https://doi.org/10.1637/00052086(2003)047[0001:OOCRIT]2.0.CO;2

CHAPMAN H. 1999. Anticoccidial drugs and their effects upon the development of immunity to Eimeria infections in poultry. Avian Pathol. 28:521-535. http://doi.org/10.1080/03079459994317 
CLARKE PL. 1979. Coccidial infection with Eimeria tenella and caecal defaecation in chicks. Br. Poult. Sci. 20:317-322. http://doi.org/10.1080/00071667908416586 COBB-Vantress Inc. 2015. Cobb 500 broiler performance and nutrition supplement. Pp. 14. http://www.cobb-vantress.com/docs/default-source/cobb-500guides/Cobb500_Broiler_Performance_And_Nutrition_Supplement.pdf

CONWAY DP, McKenzie ME. 2007. Poultry Coccidiosis Diagnostic and Testing Procedures. Third Edition. Blackwell Publishing. ISBN-13:978-0-8138-2202-0. ISBN-10: 0-8138-2202-5.

https://www.academia.edu/35924772/Poultry_Coccidiosis_Diagnostic_and_Testing_Pro cedures_THIRD_EDITION

DALLOUL RA, Lillehoj HS. 2006. Poultry coccidiosis: recent advancements in control measures and vaccine development. Expert. Rev. Vaccines 5:143-163. doi:10.1586/14760584.5.1.143. http://doi.org/10.1586/14760584.5.1.143

HAUG A, Williams R, Larsen S. 2006. Counting coccidial oocysts in chicken faeces: a comparative study of a standard McMaster technique and a new rapid method. Vet. Parasitol. 136:233-242. http://doi.org/10.1016/j.vetpar.2005.11.024

HUDMAN SP, Ketterson ED, Nolan V Jr. 2000. Effects of time of sampling on oocyst detection and effects of age and experimentally elevated testosterone on prevalence of coccidia in male dark-eyed juncos. The Auk. 117:1048-1051.

http://doi.org/10.1093/auk/117.4.1048

JOHNSON J, Reid WM. 1970. Anticoccidial drugs: lesion scoring techniques in battery and floor-pen experiments with chickens. Exp. Parasitol. 28(1):3-36. https://doi.org/10.1016/0014-4894(70)90063-9

MISOF K. 2004. Diurnal cycle of Isospora spp. oocyst shedding in Eurasian blackbirds (Turdus merula). Can. J. Zool. 82:764-768. http://doi.org/10.1139/z04-054

MASOOD S, Abbas R Z, Iqbal Z, Mansoor MK, Sindhu Z, Zia MA, Khan JA. 2013. Role of Natural Antioxidants for the Control of Coccidiosis in Poultry. Pakistan Veterinary Journal. 33(4):401-407.

https://www.researchgate.net/publication/260159600_Role_of_Natural_Antioxidants_for the_Control_of_Coccidiosis_in_Poultry

MCDOUGALD LR, Fitz-Coy SH. 2013. Coccidiosis. In: Swayne DE, Glisson JR, McDougald LR, Nolan LK, Suarez D, Nair V. (eds). Diseases of Poultry. 13th ed. WileyBlackwell Publishing, Ames, lowa, USA. Pp. 1148-1166. ISBN: 978-0-0470-95899-5. 
NAIDOO V, McGaw L, Bisschop S, Duncan N, Eloff J. 2008. The value of plant extracts with antioxidant activity in attenuating coccidiosis in broiler chickens. Veterinary Parasitology. 153(3-4):214-219. https://doi.org/10.1016/j.vetpar.2008.02.013

NRC. National Research Council. 1994. Nutrient Requirements of Poultry, ninth ed. National Academy Press: Washington, DC. USA. Pp. 176. ISBN: 978-0-309-04892-7. https://doi.org/10.17226/2114

PEEK H, Landman W. 2011. Coccidiosis in poultry: anticoccidial products, vaccines and other prevention strategies. Veterinary Quarterly. 31(3):143-161.

https://doi.org/10.1080/01652176.2011.605247

SAS. Institute Inc. 2002. SAS/STAT 9.1 User's Guide. Cary, NC. USA. ISBN 1-59047243-8.

https://support.sas.com/documentation/onlinedoc/91pdf/sasdoc_91/stat_ug_7313.pdf

SHIRLEY MW, Smith AL, Blake DP. 2007. Challenges in the successful control of the avian coccidia. Vaccine. 25(30):5540-5547. http://doi.org/10.1016/ j.vaccine.2006.12.030

SHIVARAMAIAH C, Barta JR, Hernandez-Velasco X, Téllez G, Hargis BM. 2014. Coccidiosis: recent advancements in the immunobiology of Eimeria species, preventive measures, and the importance of vaccination as a control tool against these Apicomplexan parasites. Veterinary Medicine (Auckl). 5:23-34.

http://doi.org/10.2147/VMRR.S57839

TEWARI A, Maharana B. 2011. Control of poultry coccidiosis: changing trends. Journal of Parasitic Diseases. 10-17. http://doi.org/10.1007/s12639-011-0034-7

WILLIAMS R. 2005. Intercurrent coccidiosis and necrotic enteritis of chickens: rational, integrated disease management by maintenance of gut integrity. Avian Pathol. 34:159180. http://doi.org/10.1080/03079450500112195

WILLIAMS RB. 1973. Effects of different infection rates on the oocyst production of Eimeria acervulina or Eimeria tenella in the chicken. Parasitology. 67:279-288. http://doi.org/10.1017/s0031182000046515 